\title{
Simultaneous tapering and crystallisation of silicon core optical fibres
}

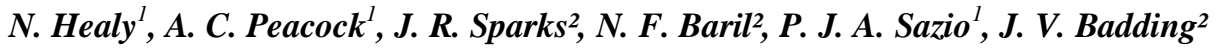 \\ 1. Optoelectronics Research Centre, University of Southampton, SO17 IBJ, UK \\ 2. Department of Materials Science, Pennsylvania State University, 16802 PA, USA
}

Silicon photonics is an increasingly active and exciting research topic that owes much to the excellent optical material properties of silicon. Crystalline silicon has a high refractive index $(\sim 3.48 @ 1.5 \mu \mathrm{m})$, a low loss transmission window between $1.2 \mu \mathrm{m}-6.7 \mu \mathrm{m}$, and a large third order optical nonlinearity (orders of magnitude greater than that of silica) making it a highly attractive material for photonic device miniaturisation. A new class of silicon waveguide, the silicon optical fibre, consists of a silicon core and a silica cladding and offers new possibilities within silicon photonics. To truly exploit the nonlinear properties of this fibre, control over its waveguiding characteristics is desirable.

Fibre tapering is a well established and convenient method of altering the guidance properties of all-silica fibre waveguides. Using a slightly modified approach to that used for conventional tapering, we provide the first demonstration of a tapered silicon optical fibre and, in turn, introduce a new degree of freedom for the design and optimisation of silicon optical fibre devices. It is envisaged that, with further work, tapering may be used to alter the fibre's waveguide dispersion and confinement parameters and also to facilitate low loss coupling between waveguides.

A high-pressure microfluidic chemical deposition technique, as described in [1], was used to fabricate the silicon optical fibre. The fibre consisted of an amorphous silicon core, with a diameter of $2.5 \mu \mathrm{m}$, and a silica cladding, with a diameter of $125 \mu \mathrm{m}$. The tapering function of a BIT communications BFF-60 arc fusion splicer was used to taper the silicon optical fibre; the arc current was set at $10 \mathrm{~mA}$, which ensured that the silicon was above its melting point of $1410^{\circ} \mathrm{C}$, and the pull distance was set so that the fibre's taper ratio was 1:2.

The tapered silicon optical fibre was examined visually using a Nikon LV100 optical microscope. Fig. 1(a) is an image of the taper's longitudinal profile, showing a smooth transition from the untapered fibre to the taper waist, where at each point along the taper the core diameter changes proportionally to the changing cladding diameter. Fig. 1(b) shows the cross-section of the silicon optical fibre both before and after (inset) tapering, from which it is evident that the tapered core has retained its original circular shape.

Raman spectroscopy was used to determine the material quality of the tapered silicon core. Fig. 1(c) shows the Raman spectra obtained for three positions along the tapered fibre: the untapered fibre, the taper transition, and the taper waist. The spectrum of the untapered fibre has a wide peak, located at $\sim 480 \mathrm{~cm}^{-1}$, that is typical of amorphous silicon. However, the spectra of both the taper transition and the taper waist have a narrow peak at $518 \mathrm{~cm}^{-1}$, confirming that the silicon core has crystallised during tapering. The broadening, and asymmetry of the tapered peaks, when compared to the reference spectrum, can be attributed to the polycrystalline nature of the core material which consists of both micro/nanocrystalline and amorphous phases. The slight red-shifting of the spectra is a result of tensile strain induced on the core at the silicon-silica boundaries when the fibre cools after tapering.
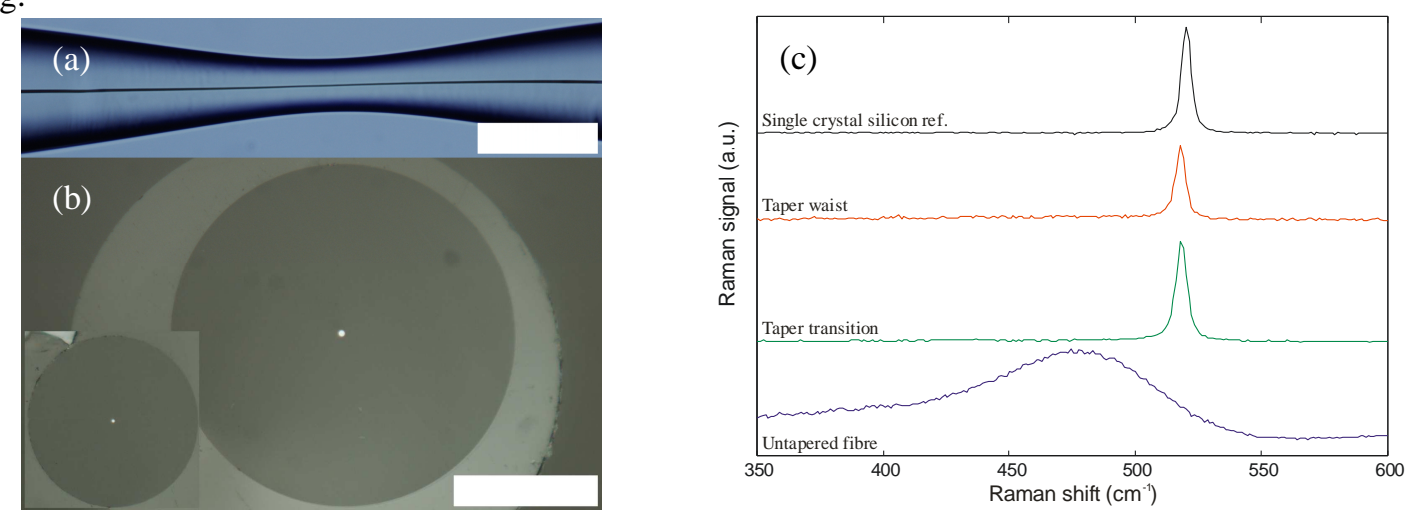

Fig. 1 Tapered silicon optical fibre: (a) Brightfield image (diascopic illumination) of the longitudinal profile, scale bar $100 \mu \mathrm{m}$. (b) Cross-sectional brightfield images (episcopic illumination) of the untapered fibre and the taper waist (inset, shown on the same scale), the scale bar is $50 \mu \mathrm{m}$. (c) Raman spectra measured at various points along the core of the tapered silicon optical fibre. Each spectrum has been normalised to its maximum.

\section{References}

[1] P. J. A. Sazio, A. Amezcua-Correa, C. E. Finlayson, J. R. Hayes, T. J. Scheidemantel, N. F. Baril, B. R. Jackson, D-J. Won, F. Zhang, E. R. Margine, V. Gopalan, V. H. Crespi, and J. V. Badding, "Microstructured Optical Fibers as High-Pressure Microfluidic Reactors," Science, 311, 1583-1586 (2006). 